This item was submitted to Loughborough's Research Repository by the author.

Items in Figshare are protected by copyright, with all rights reserved, unless otherwise indicated.

\title{
Bridging the divide between upward accountability and learning-based approaches to development evaluation: strategies for an enabling environment
}

\section{PLEASE CITE THE PUBLISHED VERSION}

http://search.informit.com.au/documentSummary;dn=513236376941726;res=IELBUS

\section{PUBLISHER}

Australasian Evaluation Society

\section{VERSION}

AM (Accepted Manuscript)

\section{PUBLISHER STATEMENT}

This work is made available according to the conditions of the Creative Commons Attribution-NonCommercialNoDerivatives 4.0 International (CC BY-NC-ND 4.0) licence. Full details of this licence are available at: https://creativecommons.org/licenses/by-nc-nd/4.0/

\section{LICENCE}

CC BY-NC-ND 4.0

\section{REPOSITORY RECORD}

Lennie, June, and Jo Tacchi. 2019. "Bridging the Divide Between Upward Accountability and Learning-based Approaches to Development Evaluation: Strategies for an Enabling Environment". figshare. https://hdl.handle.net/2134/23585. 
Bridging the divide between upward accountability and learning-based approaches to development evaluation: Strategies for an enabling environment

June Lennie and Jo Tacchi

Paper resubmitted to the Evaluation Journal of Australasia

on 12 July 2014

Text for author details:

June Lennie (left) is a Senior Research Associate in the School of Media and Communication at RMIT University, Melbourne but is based in Brisbane.

Email: j.lennie@bigpond.net.au

Jo Tacchi (right) is a Professor and Director, Research and Innovation in the School of Media and Communication at RMIT University, Melbourne.

Email: jo.tacchi@rmit.edu.au 


\title{
Bridging the divide between upward accountability and learning-based
} approaches to development evaluation: Strategies for an enabling environment

\begin{abstract}
A more complex and unstable development context and major shifts in development theory and practice over the past 20 years have created major challenges for evaluation. There remains a vast gap between the theory and practice of development evaluation that needs to be resolved. This indicates a vital need to bridge the divide between dominant resultsbased, upward accountability evaluation approaches and emergent learning, participatory and complexity-based approaches to more effectively achieve development goals. In this paper we explore this divide and contrast these approaches, highlighting some of the key challenges that each face.
\end{abstract}

Drawing on our recent research, we identify key challenges, tensions and contradictions in evaluating communication for development (C4D) that need to be better understood and addressed. We outline a new conceptual framework for evaluating C4D and other complex development initiatives as a strategy to address these challenges. We argue that achieving change requires reconceptualising accountability and learning, developing evaluation capacities that enable learning and multiple forms of accountability, and drawing on local knowledge, ideas and innovation to develop and implement more appropriate and effective evaluation approaches. These strategies can create an enabling environment in which these 
new ideas and approaches can flourish and complex development issues can be better addressed.

\section{The need for more effective and innovative approaches to development evaluation}

The vastly more complex, uncertain development context, more complex processes of change, and a number of shifts in development theory and practice over the past 20 years have created major challenges for the evaluation of development initiatives (Armytage 2011; Conlin \& Stirrat 2008; Stern at al. 2012). While new evaluation approaches have been developed and are increasingly used, there remains a vast gap between the theory and practice of development evaluation that needs to be resolved (Armytage 2011; Woodhill 2007). These major challenges and issues highlight the need for more effective, appropriate and innovative approaches to development evaluation and strategies for the creation of an enabling environment in which these new approaches can flourish and indigenous evaluation cultures and capacities can be developed (Carden 2007).

Conlin and Stirrat (2008) have identified a number of trends which pose new methodological challenges for evaluation: the rise of 'management for results' puts more stress on the impact and effects of development assistance rather than outputs and 'deliverables'; there is a move away from 'older principles of accountability and conditionality' and more focus on 'the learning function of the evaluation process' and empowerment (p. 196). In addition, there is increased stress on sector wide approaches that emphasise assisting the sector as a whole to achieve objectives that involves a range of activities, including training and capacity building, and, a growing interest in 'broader issues of development effectiveness' and the 
importance for long-term development of factors such as 'security, migration and climate change' (p. 197).

Stern at al. (2012, p.11) further highlight the complex and unstable nature of the current development context and the resultant need for new evaluation approaches. Emergency and conflict situations and the containment of terrorism has 'focussed attention on governance, peace-building and the empowerment of civil society actors'. This means that many interventions are longer term, high-risk and 'emergent' and do not suit dominant models or theories of change, making their impacts 'often difficult to evaluate or measure using established tools' (Stern 2012, p. 11).

There are clear indications of greater interest in a broader range of evaluation approaches and methodologies that can better address the complex challenges and issues in the evaluation of development initiatives. There is more focus on the value of participatory, learning and complexity-based, and constructivist approaches to evaluation, along with an growing number of critiques of results-based management and the logical framework approach (Armytage 2011; Hummelbrunner 2010; Woodhill 2007). This has led to what Armytage (2011, p. 268) calls a "'paradigm war" between positivism and constructivism'. Emphasising the disjuncture between theory and practice in development monitoring and evaluation (M\&E), Armytage (2011, p. 261) notes the 'unresolved tensions between the accountability (audit) approach and emerging effectiveness (learning) approaches'. These unresolved tensions indicate a vital need to bridge the divide between dominant resultsbased, upward accountability approaches and emergent adaptive, learning and improvement-based approaches in order to more effectively achieve development goals. It 
is increasingly recognised that linear cause-effect approaches to evaluation are ineffective for the evaluation of complex development initiatives. Instead, alternative participatory, feminist, learning and complexity-based evaluation frameworks and approaches are argued to better help development organisations and practitioners understand and address complex development issues such as poverty, gender-related inequities, and health and environmental problems (Armytage 2011; Lennie \& Tacchi 2013; Woodhill 2007).

Resolving these issues is especially important to the evaluation of communication for development (C4D). This is due to its vital role in achieving sustainable development and social change and the complexity of demonstrating the impacts of C4D using dominant evaluation approaches, a situation that has led to C4D often being under-appreciated in the development field (Lennie \& Tacchi 2013). While it has multiple meanings, C4D is generally understood to be about the use of communication in participatory processes for social change. It can involve different forms of media as well as community dialogue processes. C4D seeks to facilitate social and individual change, and to change harmful social norms. It focuses on difficult issues such as reducing gender and caste discrimination, and preventing diseases such as HIV/AIDS.

A continuous action learning and improvement-based approach to evaluation that uses a range of methods and sources of information is argued to be more effective in helping C4D and development organisations to constantly adjust and improve their initiatives in order to better meet changing community values, needs and issues and higher level development goals (Lennie \& Tacchi 2013; Woodhill 2007). This approach can foster the development of learning organisations that embed M\&E into all aspects of the organisation and program 
development cycle, take a critical approach, collaborate and share knowledge with others, and are open to learning from weaknesses and 'mistakes' (Raeside 2011).

Achieving change towards a new paradigm in development evaluation requires more understanding of the many challenges, tensions and contradictions in the current development evaluation context and reconceptualising key concepts such as 'accountability' and 'learning'. It also requires developing evaluation capacities within development organisations that enable and encourage both learning and multiple forms of accountability. This paper reviews these issues, drawing on our research into the evaluation of C4D over the past seven years, particularly findings from the Assessing Communication for Social Change (AC4SC) project which ran from 2007 to 2011 in collaboration with Equal Access Nepal, an NGO that makes radio programs focused on social change. ${ }^{1}$ We also draw on outcomes from research conducted in 2010 with an international expert panel and specialists from seven UN agencies and other bodies to develop a Resource Pack for the Research, Monitoring and Evaluation of C4D (Lennie \& Tacchi 2011).

We begin by presenting an overview and critique of the dominant upward accountability, results-based approaches to the evaluation of development initiatives. Next we provide key arguments about the value of emergent learning, participatory and complexity-based approaches to the evaluation of development and C4D initiatives whilst acknowledging their limitations. The next section outlines key challenges, tensions and contradictions in the development evaluation context and in evaluating C4D. We then consider new thinking around the concepts of accountability and learning and introduce a framework for 
evaluating C4D that offers a means of bridging the divide between upward accountability and learning-based evaluation approaches.

Finally, we note there are a range of strategies that can help to address these challenges and create the type of enabling environment that can move us towards a more empowering paradigm of development evaluation that puts people and relationships back at the centre of development. We consider evaluation capacity development as a key strategy, and briefly describe how it will be applied in a new research project designed to facilitate dialogue about these issues and build capacities in an approach to evaluating C4D that aims to be useful and feasible for both learning and accountability purposes.

\section{An overview of dominant upward accountability approaches to development evaluation}

Managing for Development Results (MfDR) is a key component of the Paris Declaration on Aid Effectiveness and reaching the Millennium Development Goals. This declaration embedded the principles of ownership, alignment, harmonisation, managing for results and mutual accountability. A key focus of these principles is demonstrating results and improving development performance. This has resulted in a greater emphasis on M\&E and evaluation capacity building in developing countries (Armytage 2011). MfDR has shifted the focus from inputs to measurable outcomes, results and impacts at all phases of the development process. It seeks to provide a framework for development effectiveness in which performance information is used for improved decision-making. MfDR is promoted as 'representing best practice in this field' and parallels the results-based management (RBM) movement (OECD 2008). 
RBM is the most commonly used technique to assess program effectiveness. It focuses on performance and the achievement of outputs, outcomes and impacts (Armytage 2011). The three principles of RBM are high levels of accountability, national ownership of results, and inclusiveness or stakeholder engagement (UNDG 2011). Key elements of RBM include identifying measurable results, selecting indicators to measure progress, setting targets, developing data collection systems, comparing results to targets, and using performance information to make decisions, learn what works and what doesn't and report to stakeholders (http://www.mango.org.uk/guide/whyrbmnotwork). This system is usually summarised in a logical framework grid, which is a central tool of MfDR (Armytage 2011).

Since the 1970s the logical framework approach (LFA) has come to play a central role in planning, monitoring, evaluating and managing international aid and development interventions (Hummelbrunner 2010). The LFA is one of a larger class of tools known as program logic models that are commonly used in project cycle management and program evaluation.

\section{Critiques of dominant approaches to development evaluation}

In recent years there has been an increasing number of critiques of RBM and LFA. These critiques have particular implications for the evaluation of complex development initiatives such as C4D programs which seek to influence behaviour and social change. A number of the issues raised below also emerged in our recent research projects.

Dominant development evaluation approaches such as LFA were developed in the West and are often imposed on development organisations by donors who tend to see the logframe as 
'something definite against which aid recipients can be held accountable' (Hummelbrunner 2010, p. 4). However, logframes are often not used beyond the initial planning process because they 'do not fit with how NGO work really happens on the ground' (http://www.mango.org.uk/guide/whyrbmnotwork).

The LFA was originally designed from a strong central authority and control perspective that assumes people 'operate in rather stable environments' (Hummelbrunner 2010, p. 2). Ramalingam (2011, p. 3) points out that many 'modern management' approaches such as RBM are underpinned by an engineering theory that takes 'a reductionist approach to problem solving'. However, he notes that 'Even in the best case scenario, such approaches can only be followed loosely because real-world systems cannot be divided up and controlled in neat and tidy ways' (Ramalingam 2011, p. 3). The LFA and results-based approaches therefore tend to stifle participation since they 'reinforce relationships of power and control ... [embodying] a linear logic associated with things rather than people' (Chambers \& Pettit 2004, p. 145). An important weakness of the LFA in non-Western contexts is that it does not allow space for styles of communication and working that are more appropriate than Western styles (Marsden 2004).

While logic models have several strengths and are useful in evaluations that are focused on simple problems, they have been widely criticised as inflexible and unable to capture change in complex, dynamic contexts. They are bad at accommodating local culture, or capturing unexpected or emergent outcomes or change. Like all models, the logframe represents the simplification of what are often quite complex social processes. It also avoids the importance of process. Patton (2011, p. 18) comments that linear logic models work well 'in simple 
situations of high certainty and high agreement about what to do', but less well in complex and dynamic situations where the development goal or the social change is less easily defined. These approaches can have a distorting effect when change is 'emergent, evolving and adapting' (Patton 2011, p. 18).

These critiques highlight that linear cause-effect approaches to evaluating social change initiatives are too simplistic and focused too much on pre-planning, developing pre-set indicators, measurement, and proving impacts rather than on better understanding the local context, the process of change, why and how change happens, and what can be learned from this in order to design more effective initiatives or improve existing initiatives. They have also been seen as stifling the innovation and flexibility required to achieve outcomes (Ramalingam 2011, p. 6).

Results-based approaches encourage a narrow focus on projects and programs rather than on the complex systems, relationships and constantly changing contexts in which development and change takes place. Consideration of context in evaluation is even more important in developing countries. This is due to the more vulnerable and unpredictable nature of societies and institutional systems, and issues such as dependence on 'uncontrollable forces', high levels of poverty, lack of 'resources, opportunities, and exposure to new ideas', and the domination of those who are already powerful (Ofir \& Kumar 2013, p. 14). It is increasingly recognised that, as Woodhill $(2007$, p. 89) points out, 'it is the relationships between different actors and the ways these relationships are facilitated and supported that ultimately determines what will be achieved' in development projects. 
MfDR can often have the 'perverse consequence' (Armytage 2011, p. 268) of encouraging a focus on less important aspects of an initiative and more visible, short-term changes that are not good indicators of long-term social change. It is well understood that social change usually takes a long time to occur yet evaluations of development initiatives are often expected to show outcomes and impacts after only a few years. Natsios $(2010$, p. 1$)$ makes the point that a central principle of development theory is that 'those development programs that are most precisely and easily measured are the least transformational and those programs that are most transformational are the least measurable'.

Jakimow (2008, p. 315) notes a shift from linear, outcomes-based understandings of knowledge in the development sector 'to one that requires a systematic understanding of the complex influences and contexts that shape it. Knowledge should be localized and contextualized, made relevant and reinvented by the "local people"'. However, highlighting the 'disjuncture between rhetoric and practice' in 'reflexive development', Jakimow (2008, p. 318) cites evidence that 'institutional conditions prevent the incorporation of multiple voices, or the ability to pursue multiple approaches'. She notes that development agencies maintain a top down RBM approach that does not provide space for local knowledge.

Dominant approaches to development evaluation are more often focused on meeting the expectations and accountability requirements of donors rather than being accountable to all of the stakeholders involved and encouraging everyone to learn from the process in order to improve programs, develop better capacities and put more effective strategies into practice. While they claim to encourage learning, in practice this is often not the case. 


\section{Learning, participatory and complexity-based evaluation approaches}

Given all of the above, there is growing interest in the value of emergent learning, participatory and complexity-based approaches to the evaluation of development and C4D initiatives, as an alternative. These approaches are underpinned by values, principles and practices that strongly align with those of C4D. However, these approaches also present challenges and issues that need to be taken into account.

\section{Learning-based approaches}

Conlin and Stirrat (2008, p. 196) have identified an increasing stress on 'the learning function of the evaluation process'. A continuous learning-based approach to M\&E and organisational development that uses various methods and sources of information and involves developing positive visions of change and success is seen as more effective in helping organisations to constantly adapt and improve their initiatives and ways of working in order to better meet community needs and development objectives (Lennie \& Tacchi 2013; Pearson 2011; Woodhill 2007). This has significant implications for evaluation capacity development and requires a 'significant paradigm shift' (Woodhill 2007).

The learning-based component of our framework for evaluating C4D that we outline later in this paper is based on action learning and participatory action research (PAR) principles and processes that aim to achieve good communication, cooperation, collaboration and trust between those involved. The aim is to facilitate and encourage continuous learning, mutual understanding, empowerment, creative ideas and thinking, and responsiveness to new ideas and different attitudes, values and knowledge. This involves evaluation being fully integrated into organisations and the whole program cycle and a diversity of staff, stakeholders and 
community members taking responsibility for research and evaluation activities. We argue this helps to develop the wide range of evaluation capacities that are required in this approach (Lennie \& Tacchi 2013 p. 23-24).

This approach can foster the creation of learning organisations that value $M \& E$, share ideas, knowledge and expertise with others, engage in regular critical reflection in order to learn from experience, successes and mistakes, improve their practices, respond effectively to complex and rapidly changing contexts, and incorporate local innovation and ideas in the process (Pearson 2011; Raeside 2011). This type of learning can have a greater impact than individual learning as it 'involves intentional learning throughout various areas and levels of the organisation, which then interact to create a strong and effective learning foundation' (McCoy, Rose \& Connolly 2013, p. 17). However, as Guijt (2010, p. 277) points out, 'Official policies that profess the importance of learning are often contradicted by bureaucratic protocols and accounting systems which demand proof of results against pre-set targets. In the process, data are distorted ... and learning is aborted'.

Woodhill (2007 p. 83) suggests that the challenge in building a learning-oriented M\&E paradigm is 'to use effective reflective processes that can capture and use actors' wealth of tacit knowledge that is all too often ignored'. Carden $(2007$, p. 53) makes the case for development evaluation being 'best done by locally based researchers and organizations who know the culture and context ... and have a responsibility to build capacity to use research in decision-making in local institutions, governmental, corporate or nongovernmental'. There is a need to better appreciate locally developed learning and evaluation methods and more appropriate and effective evaluation methodologies that have 
been developed and tested with people in the development context. The AC4SC project aimed to develop such a methodology through a PAR approach to the development of a participatory monitoring and evaluation (PM\&E) methodology for Equal Access Nepal (EAN) which included the development and training of a network of community researchers. Over four years we worked with EAN to collaboratively develop, test and refine the methodology and each component, which resulted in the production of a transferable toolkit. ${ }^{2}$

However, learning-based approaches can be a challenge to implement in development contexts such as Nepal where people are often discouraged from being critical of teachers and those in authority. We found that rigid learning systems and the hierarchical, castebased culture led to problems with using participatory processes in the AC4SC project. For example, while we wanted to work through issues and problems and collaboratively develop approaches that worked in the context, EAN staff were often frustrated because we would not simply tell them what to do. This indicated that they considered us the 'experts', despite our insistence that we were facilitators and they were the people with the relevant knowledge and contextual understanding (Tacchi, Lennie \& Wilmore 2013). Pearson (2011) encountered similar issues with implementing her creative capacity building activities in a Cambodian NGO in which an action learning approach was used. She found that people were resistant to change and fearful of exploring or stirring emotional depths, given their recent history. However, the creative and innovative approaches she used encouraged different ways of thinking and highlighted the different understandings of what was expected in learning situations and what constitutes learning among the Cambodian staff of the NGO. 


\section{Participatory and mixed methods approaches}

There has long been an appreciation of the value of participatory approaches to the evaluation of development and C4D initiatives (Estrella et al. 2000; Lennie \& Tacchi 2013). Our research and that of others has found that participatory evaluation is effective in developing research and evaluation capacities and more realistic indicators of social change, providing more honest and reliable data and identifying unexpected or unintended impacts of programs and a range of other benefits.

However, in using participatory approaches, there is a need to be alert to power dynamics and issues of inclusion and exclusion, empowerment and disempowerment. These approaches also require considerable skills and capacity development and take time and resources to use effectively. Given other demands on staff and stakeholders, achieving a high level of participation and commitment to the evaluation process is not always easy. In particular, it is often difficult for development interventions to reach the most marginalised groups such as the very poor.

The use of mixed methods is also now considered by many as the optimal way to undertake development evaluation (Donaldson, Azzam \& Conner 2013) and is a key feature of our framework for evaluating C4D. Stern et al $(2012$, p. 81$)$ note that a mixed methods approach is now 'commonplace'. A rigorous mixed methods approach can provide a fuller and more realistic picture of change that is able to capture the voices, concerns and values of diverse stakeholders (Bamberger, Rugh \& Mabry 2006). 


\section{Systems and complexity-based approaches}

A holistic perspective based on systems thinking, complexity theory and action research is increasingly seen as important to better understand and address complex development problems (Burns 2007; Ramalingam et al. 2008). From this perspective, the social systems within which development and C4D are implemented are viewed as processes that are dynamic, historical and capable of continuous transformation and change in ways that cannot always be predicted. This emphasises the need to understand how the wider systems, networks, inter-relationships, boundaries, and other aspects of the context in which C4D is implemented actually operate, and how they can influence outcomes (Lennie \& Tacchi 2013).

Sensitivity to contextual factors, organisational norms and societal values is critical in systems-oriented evaluations (Patton, 2011, p. 120) such as those using Outcome Mapping methodology. The critical reflection, problem solving and action learning skills that are required in systems approaches are increasingly considered important to the effective, ongoing evaluation of development initiatives.

Some of the characteristics of complexity theory and systems thinking have fundamental similarities to participatory monitoring and evaluation (PM\&E):

1. A shift in focus to interrelationships and processes rather than snapshots, seriously challenging dominant linear explanations of systemic phenomena.

2. An understanding of development as complex, emergent and transformative.

3. A shift to the bigger picture and interconnections, with much focus on boundaries and the values they reflect. (Byrne, 2008, p. 9, based on Williams \& Iman, 2006). 
There is growing appreciation of the 'simple, complicated, complex' typology (Patton 2011) to guide the evaluation of development initiatives. This helps us to understand the difference between the diverse kinds of problems that need to be addressed in the development context and to better match the intervention or evaluation approach to the nature of the situation. Both complicated and complex aspects of interventions present challenges for traditional linear approaches to M\&E (Rogers 2009). Using this typology to inform the evaluation of C4D requires considering the multiple possible paths to achieving impacts, and what other factors contributed to achieving results, including whether the context was favourable or otherwise (Rogers 2009).

While systems and complexity-based evaluation approaches and methodologies have many strengths, and are a key aspect of our framework for evaluating C4D, they also have some limitations and weaknesses. For example, our UN consultations identified some issues with the complexity, 'overly detailed' and time-consuming nature of the Outcome Mapping methodology and difficulties with its terminology. As with participatory evaluation, some specialised skills are also needed to effectively undertake systems and complexity-based evaluation.

\section{Challenges, tensions and contradictions}

Key challenges, tensions and contradictions in evaluating C4D that we identified in our research include those related to:

- Contextual and institutional challenges

- Demonstrating the impact of C4D

- Developing and sustaining evaluation capacities 
- Inadequate funding, time and other resources

- Policies and attitudes to alternative evaluation approaches

\section{Contextual and institutional challenges}

As we have noted, there are considerable challenges and issues in conducting evaluations in developing countries with significant social, economic and political problems, as well as challenges related to meeting the accountability requirements of donors and organisational challenges and isues. There are also issues related to geographic and cultural barriers, local political issues, and difficulties with travel and communication. An analysis of nine in-depth interviews with a variety of EAN staff involved in the AC4SC project identified a wide range of challenges, issues and barriers that affected the ability of those involved in the project to effectively reach its aims. A key challenge was the complex and unstable political situation in Nepal which affected mobility and security and was seen as promoting negative thinking in the community. In addition, lack of reliable electricity supply slowed the process of coding and analysing qualitative research and evaluation data and prevented EAN from following plans and meeting deadlines.

\section{Demonstrating the impact of C4D}

Demonstrating the impact of C4D is quite difficult compared with other development initiatives where it can be easier to track and isolate changes due to a particular program; for example, assessing changes in diseases rates due to a polio eradication program. With C4D, a complex network of effects is often at play due to factors such as the mediation of mass media messages through interpersonal and group communication (Inayaki 2007). This means 
that evaluators experience particular problems with attribution, especially when multiple partnerships and strategies are involved in trying to bring about social change over long periods of time. In addition, there is often pressure to demonstrate impacts using inappropriate methods that limit the ability to understand social and behaviour change (Lennie \& Tacchi 2013).

Several EAN interviewees identified that it was difficult to directly attribute changes to the radio programs alone and to 'prove' the extent of social change that could be attributed to their programs. They commented that multiple factors affected change and there were many external factors beyond their control that could affect change, including the constant process of internal migration and changes in communication and information channels. Social change was considered difficult to bring about, and resistance to change was seen as largely due to the deeply rooted traditional culture in many parts of Nepal.

\section{Developing and sustaining evaluation capacities}

Our research has identified a lack of evaluation capacity at all levels, especially in approaches that are more effective for evaluating C4D, and lack of opportunities for ongoing capacity development, training and support. A wide range of skills are needed to effectively use participatory and complexity-based evaluation approaches and methodologies. However, developing, implementing and sustaining evaluation capacity development (ECD) presents particularly difficult challenges and issues for time, skill and resource-poor organisations in developing countries. Responses about this issue from an online survey undertaken for our UN Resource Pack consultations included: 
Few skilled practitioners in many countries to conduct research, monitoring and evaluation of C4D.

Weak or non-existent expertise of personnel within the national agency(ies) with whom the UN agency is working.

Weak capacity for research and evaluation, especially at organisational levels, and inadequate resources to strengthen capacity at all levels, over a realistic timeframe.

Some EAN interviewees thought the high turnover of M\&E Coordinators in the two years since the AC4SC project began had led to a lack of consistent leadership and capacity in the M\&E team and created problems with the continuity of staff and project activities. The ongoing participatory, ethnographic-type research approach being used by the community researcher (CR) network was considered by some interviewees as an important means of documenting and observing the process of gradual change in a community. However, a key challenge was improving the capacity of the CRs to obtain better quality and more useful data that focused more on the radio programs and their impacts and less on community needs and issues.

\section{Inadequate funding, time and other resources}

Our research also identified issues with inadequate funding and other resources such as time for research and evaluation of C4D, which was seen by some as a low priority. While longitudinal studies are considered most effective, they are often hard to fund. Responses about this issue from our UN Resource Pack consultations included: 
Resources needed for research, if available (which they are usually not) would be disproportionate to the scale of the project/programme.

Under resourcing the effort, expecting impact results from what is really just 'a drop in the ocean' case study.

Lack of time, human and financial resources and capacity to fully engage with the AC4SC project and undertake project-related activities was a further challenge identified in the EAN interviews. A senior management member reported that donors often tried to reduce the amount of funding available for M\&E, restricting its effective implementation.

\section{Policies and attitudes to alternative evaluation approaches}

Our consultations for the UN Resource Pack also found that funders and evaluation managers often saw alternative evaluation approaches and methodologies as less valid, objective or rigorous than dominant or standard results-based approaches. Responses about this issue included:

The apparent obsession with methods and tools, to the neglect of deeper, fundamental questions like: Who is the evaluation for? What is it for? Who are the intended users of the evaluation? What are the intended uses? How will the process itself empower those involved and strengthen wider communication for development processes?

Too much jargon and mystification of the process, lack of simplicity and lack of clarity about what is being evaluated. 
A senior EAN management member suggested that one of the key challenges of the AC4SC project was breaking away from traditional M\&E processes and the 'traditional mindset of baseline or endline, 10 focus group discussions, 20 key informant interviews, and that's the $M \& E^{\prime}$, which takes time to change.

\section{Bridging the divide between accountability and learning-based evaluation approaches}

Recent literature has put forward new ideas about the concepts of 'accountability' and 'learning'. This suggests a need to rethink these concepts and the inter-relationships that have been identified between them. There is also a growing emphasis on the need to think about multiple forms of accountability - upward, downward, internal and personal.

Cracknell (2000) suggests that the aims of accountability and learning are incompatible in the evaluation of development aid programs. However, Guijt (2010) argues that there is no inherent contradiction between them, highlighting the synergies and the overlaps in capacities that are needed for both under complex conditions. Guijt (2010, p. 280-281) suggests that power dynamics are a key to breaking the status quo and that resolving the tensions 'requires a new ideas set, not just changes in organizational systems and practices'. In an attempt to change the perception of evaluation as a threat, Feinstien (2012, p. 103) makes a case for an evaluation approach in which 'accountability complements the role of evaluation as a learning tool' and argues that 'accountability provides an incentive for learning'. He suggests that this is facilitated by 'the development of a learning culture where mistakes are seen as opportunities for learning and learning as an important source for growth and development'. 
Over the past decade there has been a shift from evaluations being mainly focussed on upwards, external accountability to donors, to a greater focus on internal, personal and downwards accountability to primary stakeholders, accompanied by experimentation with 'participatory approaches that address issues of power, justice and rights and open up new frontiers of enquiry, learning and understanding of change' (David and Mancini 2011, p. 245). Jones (2011, p. ix) observes the emergence of innovative systems for feedback and increasing emphasis on transparency and accountability in development interventions. Conlin and Stirrat (2008, p. 202) also note the changing ways in which accountability is defined: 'No more is it simply a matter of accountability to donors, but also of downward accountability to beneficiaries'. A participant in a recent international 'Big Push Forward' event suggested that accountability should be re-framed to be about 'demonstrating an organisation's continuous learning and adaptation while recognising that change is often an effect of collaboration between many partners and that it is rare to attribute results to just one organisation' (Eyben, 2012).

A growing number of initiatives have emerged that aim to develop better accountability to communities and stakeholders. Jones (2011, p. 27) cites Action Aid's Accountability, Learning and Planning System (ALPS), which stimulates ongoing change in Action Aid's 'planning, strategy, appraisals, annual reports and strategic reviews, bringing them more in line with principles of downward accountability'. Another example provided by David and Mancini (2011, p. 246) are the processes and mechanisms developed by Oxfam Australia and Oxfam New Zealand, which include complaints mechanisms, annual reflection processes, stakeholder surveys and processes for transparent sharing of analysis and feedback with stakeholders. 


\section{A new conceptual framework for evaluating C4D}

We suggest that a key strategy in moving beyond these tensions and paradoxes is to draw upon a new conceptual framework for evaluating C4D and other complex development initiatives (Lennie \& Tacchi 2013; Tacchi \& Lennie 2014). In response to the main challenges and issues outlined above, the framework is made up of seven inter-related components: participatory, holistic, complex, critical, emergent, realistic and learning-based, which are each underpinned by a set of principles, as shown in Figure 1.

\section{FIGURE 1: KEY COMPONENTS AND CONCEPTS IN THE FRAMEWORK FOR EVALUATING C4D}

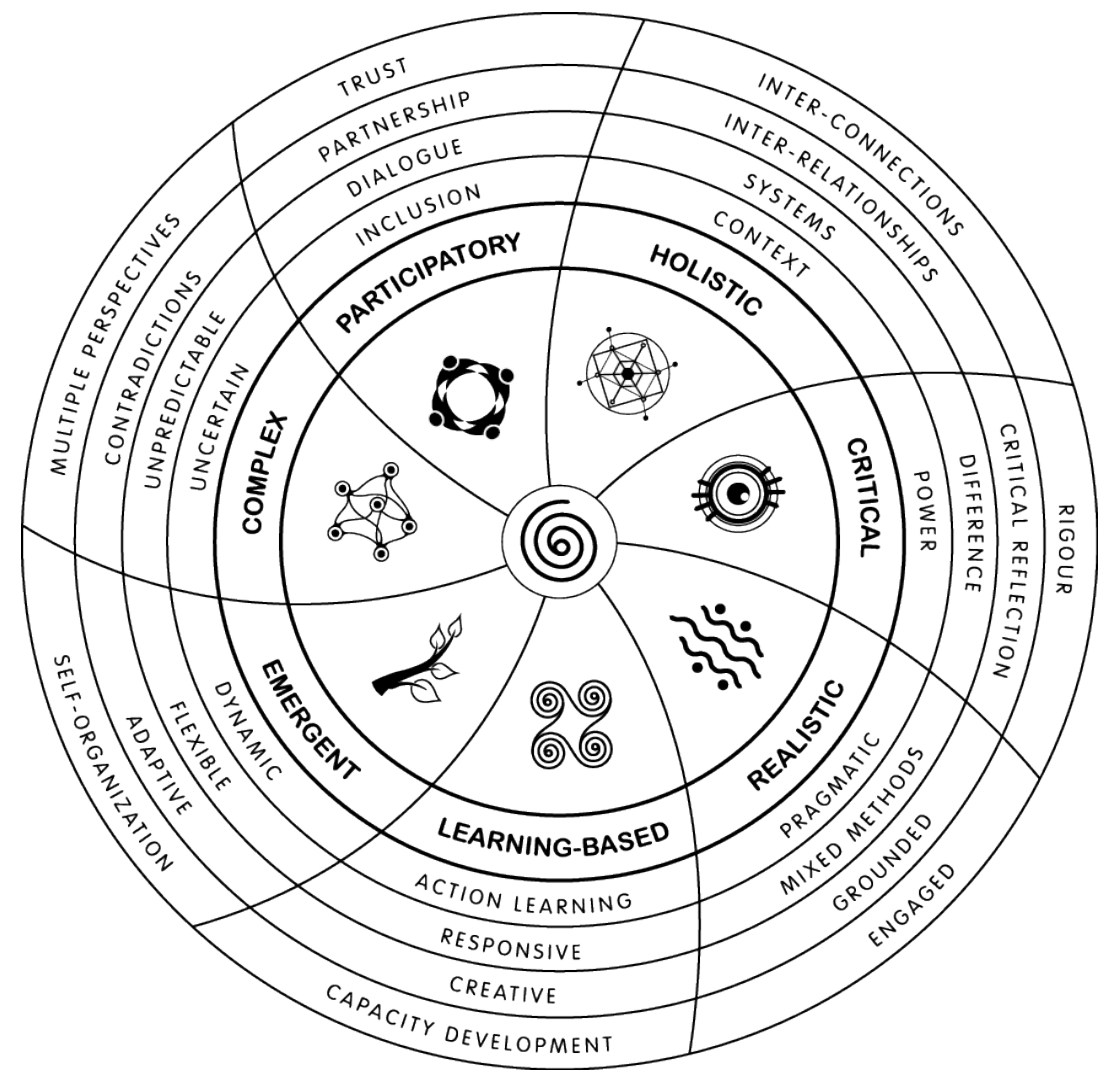

Effectively evaluating C4D requires a shift in focus from the impact of interventions to changes in wider social and organisational systems and relationships. Our framework sees 
social change as a process that is complex, non-linear, emergent, dynamic, and often contradictory. In complex systems social change happens through multi-level, interconnected and unpredictable relationships and processes. Guijt (2010, p. 287) suggests that the complex domain offers a 'liberating idea' about the process of change and 'is the domain where accountability and learning depend on each other'. Our framework allows for complexity and does not attempt to oversimplify or assume a cause and effect linear model of change. It insists on locating actions and evaluations within local contexts to help to ensure realistic, emergent and pragmatic processes. It calls for a critical reflection to understand power dynamics and to learn from weaknesses, failures and mistakes as well as success. It places activities and evaluation within relevant systems, structures and relationships, and advocates participatory and dialogic approaches to engage stakeholders and recognize different knowledge, expertise and perspectives and experience.

As Burns (2007) points out, there is an urgent need to pay attention to social norms in order to win community support, without which development interventions will not be sustainable. Clearly, the evaluation of social change initiatives requires a high level of awareness of gender issues. However, while the participation of women is a fundamental principle for development, it is often difficult to carry through because of gender inequalities in many societies.

The framework emphasises people, relationships, processes, principles and values, particularly participation, inclusion, open communication, trust, continuous learning and powerful listening. When it is well-planned and facilitated, this approach enables the inclusion of diverse perspectives in an evaluation, and can have significant effects in terms of 
the empowerment, inclusion, and capacity development of participants and stakeholders. It aims to encourage critical questioning and new ways of thinking about the fundamental theories of development and dominant approaches to evaluating development initiatives. The framework can therefore help to increase understanding of the important contributions of C4D and evaluation to the process of development and social change. It can help to develop, strengthen and improve C4D initiatives and C4D organisations, and to suggest strategies and guidance towards more effective and appropriate approaches to evaluating C4D.

\section{Effective strategies for an enabling environment}

There are a range of other strategies to help address the challenges and issues we have identified and to create the type of enabling environment that can move us towards a more empowering paradigm of development evaluation that puts people and relationships back at the centre of development. Here we focus on building evaluation capacities that enable both learning and accountability and empowering C4D organisations, M\&E staff and communities to develop and implement more appropriate, effective and innovative evaluation methods.

\section{Building evaluation capacities that enable both learning and accountability: There is a}

significant need to build capacities in evaluation and impact assessment approaches that are more effective and appropriate in the long-term for evaluating C4D than dominant or standardised approaches. New approaches such as outcome mapping and developmental evaluation, along with well established approaches such as PM\&E, can help development organisations to become learning organisations. One strategy to resolve the tension between accountability and learning, suggested by Guijt (2010: 289), is to creatively merge 
needs such as 'upward accountability requirements with strategic accountability and organisational learning needs'. This means taking a holistic, long-term, learning-centred approach to evaluation capacity development (ECD) that includes the use of participatory methodologies (Horton et al. 2003; Lennie \& Tacchi 2013). It entails embedding ECD into C4D practice, tapping into local knowledge, and increasing appreciation of the important role of evaluation in C4D. The effective development of learning cultures in development organisations requires good communication, cooperation, collaboration and trust between evaluators and others, and the integration of evaluation into the whole project or program cycle.

\section{Empowering C4D organisations, M\&E staff and communities to develop and implement more appropriate, effective and innovative evaluation methods}

The development of flexible, community-based research and evaluation approaches and the long history of the use of PAR in the development field highlights the need to look to local knowledge, ideas and innovation in order to develop more appropriate, effective and innovative evaluation approaches and methods. This should help to more effectively and sustainably improve evaluation capacities in C4D and development organisations. As Hay (2010, p. 229) points out: 'Instead of looking to the north for curriculum and methods, [evaluation] field building entails experimentation and indigenous innovation, building on the best ideas available but creating something better'. Hay (2010, p. 229) further suggests that the on the ground experience of the many thousands of people involved in learning from innovative and locally contextualised development work 'can help stimulate evaluation theory, methods, and applications within a framework of use and practice'. Our 
consultations to develop the UN Resource Pack on R,M\&E in C4D highlighted a need for more freedom and flexibility in the selection and use of evaluation approaches, methodologies and methods so that evaluations are appropriate to the particular local context, participants and program.

\section{Bridging the divide between adaptive and results-based management approaches}

Our framework for evaluating C4D is being implemented in a new three year collaborative research project Evaluating Communication for Development: Supporting Adaptive and Accountable Development that will be conducted by RMIT, UNICEF, the Eidos Institute and the University of Hyderabad between 2014 and 2017. The main aim of the project is to develop and apply an approach to evaluating C4D that is useful and feasible for both learning and accountability purposes.

The project will be conducted in various countries in the Asia-Pacific region, using PAR and participatory evaluation methodologies, based on findings and experiences from previous projects such as AC4SC, out of which the framework was developed. The project aims to build evaluation capacity in regional knowledge hubs, establish virtual learning networks and other activities that enable ongoing dialogue and learning among the groups involved (Jakimow 2008) and to understand how this contributes to sustainable development and social change. These activities will enable sharing of knowledge and experiences in order to continuously improve effectiveness. The project will build on existing ECD initiatives in the regions involved, including activities by UNICEF, and regional evaluation associations. It will link, through UNICEF and BetterEvaluation, to the EvalPartners initiative which aims to 
strengthen the evaluation capacities of civil society organisations and evaluation associations around the world.

\section{Conclusion}

Achieving change towards a new paradigm in development evaluation that puts participation, continuous action learning and relationships back at the centre of development requires better consideration of the many challenges, tensions and contradictions in the current development evaluation context that prevent the adoption of more appropriate approaches. It requires reconceptualising key concepts such as accountability and learning; developing evaluation capacities that enable both learning and multiple forms of accountability; and empowering local development actors to draw on their knowledge and ideas to design and implement more appropriate and effective evaluation approaches. This can foster the creation of learning organisations that value evaluation, share knowledge with others, engage in regular critical reflection to learn from experience, continually improve their practices, and can respond more effectively to complex and rapidly changing contexts.

We have outlined a range of strategies that can help to move us towards a greater understanding and use of alternative participatory, learning and complexity-based evaluation approaches. We argue that this can lead to more effective and appropriate evaluation of complex development and C4D initiatives. Implementing these strategies can contribute to better achieving development and social change goals. The new Evaluating C4D project aims to test our new framework, develop new knowledge and implement innovative strategies that will help to achieve these aspirations. 
We propose that the development of an enabling environment is critical for an evaluation framework that focuses on participation and learning, with all of the challenges this brings in the development context. Building effective capacities in evaluation is essential to the success of this new evaluation framework.

\section{Notes}

1. The AC4SC project was undertaken by the authors in collaboration with Andrew Skuse and Mike Wilmore from the University of Adelaide and funded by the Australian Research Council and Equal Access.

2. This methodology underpins the Equal Access Participatory Monitoring and Evaluation Toolkit which can be found at http://betterevaluation.org/toolkits/equal_access_participatory_monitoring.

\section{References}

Armytage, L 2011, 'Evaluating aid: an adolescent domain of practice', Evaluation, vol. 17, no. 3, pp. 261-276.

Balit, S 2010, 'Communicating with decision makers', Glocal Times, issue 14, pp. 1-8.

Bamberger, M, Rugh, J \& Mabry, L 2006, Real world evaluation, Sage, Thousand Oaks.

Burns, D 2007, Systemic action research: a strategy for whole system change, The Policy Press, Bristol.

Byrne, A 2008, 'Evaluating social change and communication for social change: new perspectives', MAZI, issue 17, pp. 1-14. 
Carden, F 2007, 'The real evaluation gap', Alliance, vol. 12, no. 4, pp. 53-54.

Chambers, R \& Petit, J 2004, 'Shifting power to make a difference', in L Groves and R Hinton (eds) Inclusive aid: changing power and relationships in international development, Earthscan, London.

Conlin, S \& Stirrat, R 2008, 'Current challenges in development evaluation', Evaluation, vol. 14, no. 2, pp. 193-208.

Cracknell, B 2000, Evaluating development aid: issues, problems and solutions, Sage, Thousand Oaks.

David, R \& Mancini, A 2011, 'Participation, learning and accountability: the role of the activist academic', in A Cornwall \& I Scoones (eds), Revolutionizing development: reflections on the work of Robert Chambers, Earthscan, London, pp. 241-248.

Donaldson, S, Azzam, T \& Conner, R 2013, 'Future directions for improving international development evaluations' in S Donaldson, T Azzam \& R Conner (eds), Emerging practices in international development evaluation, Information Age Publishing, Charlotte, NC, pp. 225231.

Estrella, M with Blauert, J, Campilan, D, Gonsalves, J, Guijt, I, Johnson, D et al. (eds) 2000, Learning from change: issues and experiences in participatory monitoring and evaluation, Intermediate Technology Publications, London, viewed 11 July 2014, <http://www.idrc.ca/openebooks/895-3/\#page_201>. 
Eyben, R. 2012, 'If they write a cheque today, they want a result tomorrow': Big Push Forward in New York, viewed 16 October 2012, <http://bigpushforward.net/archives/1786>. Eyben, R 2013, Uncovering the politics of 'evidence' and 'results'. A framing paper for development practitioners, viewed 15 November 2013 <http://bigpushforward.net/wpcontent/uploads/2011/01/The-politics-of-evidence-11-April-20133.pdf>.

Feinstein, O 2012, 'Evaluation as a learning tool', in S Kushner \& E Rotondo (eds) Evaluation voices from Latin America. New Directions for Evaluation, vol. 134, pp. 103-112.

Guijt, I 2010, 'Exploding the myth of incompatibility between accountability and learning'. In J Ubels, N-A Acquaye-Baddoo \& A Fowler (eds) Capacity development in practice, Earthscan, London, pp. 277-291.

Hay, K 2010, 'Evaluation field building in South Asia: reflections, anecdotes, and questions', American Journal of Evaluation, vo. 31, no. 2, pp. 222-231.

Horton, D, Alexaki, A, Bennett-Lartey, S Brice, K N, Campilan, D, Carden, F et al 2003, Evaluating capacity development: experiences from research and development organizations around the world, International Service for National Agricultural Research, The Hague. Hummelbrunner, R 2010, 'Beyond logframe: Critique, variations and alternative', in N Fujita (ed) Beyond logframe; Using systems concepts in evaluation, Foundation for Advanced Studies on International Development, Tokyo, pp. 1-33.

Inagaki, N 2007, Communicating the impact of communication for development. Recent trends in empirical research, Working Paper Series no. 120, World Bank, Washington DC. 
Jakimow, T 2008, 'Answering the critics: the potential and limitations of the knowledge agenda as a practical response to post-development critiques', Progress in Development Studies, vol. 8, no. 4, pp. 311-323.

Jones, H 2011, Taking responsibility for complexity: How implementation can achieve results in the face of complex problems, ODI Working Papers 330, Overseas Development Institute, London, viewed 15 November 2013, <http://www.odi.org.uk/resources/details.asp?id=5275\&title=complex-problemscomplexity-implementation-policy>.

Lennie, J. and Tacchi, J. 2011, Researching, monitoring and evaluating communication for development: Trends, challenges and approaches, Report on a literature review and consultations with Expert Reference Group and UN Focal Points on C4D. Prepared for the United Nations Inter-agency Group on Communication for Development, UNICEF, New York, viewed 11 July 2014, <http://www.unicef.org/cbsc/files/RME-RP-

Evaluating_C4D_Trends_Challenges_Approaches_Final-2011.pdf >.

Lennie, J \& Tacchi, J 2013, Evaluating communication for development: a framework for social change, Abingdon, Routledge.

Marsden, R 2004, 'Exploring power and relationships: A perspective from Nepal', in L Groves and R Hinton (eds) Inclusive aid: changing power and relationships in international development, Earthscan, London. 
McCoy, A, Rose, D \& Connolly M 2013, 'Developing evaluation cultures in human service organisations', Evaluation Journal of Australasia, vol. 13, no. 1, pp. 15-20.

Natsios, A, 2010, 'The clash of the counter-bureaucracy and development', Centre for Global Development Essay, viewed 10 July 2014, <http://www.cgdev.org/publication/clashcounter-bureaucracy-and-development>.

OECD 2008, Managing for development results Information Sheet, viewed 10 July 2014, <http://www.mfdr.org/About/Final-MfDR-information-sheet.pdf>.

Ofir, Z \& Kumar, AK 2013, 'Evaluation in developing countries. What makes it different?' in S Donaldson, T Azzam \& R Conner (eds), Emerging practices in international development evaluation, Information Age Publishing, Charlotte, NC, pp. 11-24.

Patton, MQ 2011, Developmental evaluation: applying complexity concepts to enhance innovation and use, Guilford Press, New York.

Pearson, J 2011, Creative capacity development: learning to adapt in development practice, Kumarian Press, Sterling VA.

Raeside, A 2011, 'Are INGOs brave enough to become learning organisations?', in H Ashley, N Kenton \& N Milligan (eds) How wide are the ripples? From local participation to international organisational learning. Participatory Learning and Action, vol. 63, pp. 97-102.

Ramalingam, B 2011, 'Why the results agenda doesn't need results, and what to do about it', viewed 10 July 2014, <http://aidontheedge.info/2011/01/31/why-the-results-agendadoesnt-need-results-and-what-to-do-about-it/>. 
Ramalingam, B \& Jones, H with Reba, T \& Young, J 2008, Exploring the science of complexity: ideas and implications for development and humanitarian efforts, ODI Working Paper 2nd ed., London: Overseas Development Institute, viewed 21 March 2012, <http://www.odi.org.uk/resources/download/583.pdf>.

Rogers, P. 2009, 'Matching impact evaluation designs to the nature of the intervention and the purpose of the evaluation', in $\mathrm{H}$ White (ed) Designing impact evaluations: different perspectives, Working Paper 4, The International Initiative for Impact Evaluation, New Delhi, viewed 15 November 2013, <http://www.3ieimpact.org/en/evaluation/workingpapers/working-paper-4/>.

Stern, E, Stame, N, Mayne, J, Forss, K, Davies, R \& Befani, B 2012, Broadening the range of designs and methods for impact evaluation. DFID Working Paper 38. DFID, London.

Tacchi, J, Lennie, J \& Wilmore M, 2013 'Critical reflections on the use of participatory methodologies to build evaluation capacities in international development organisations'. In S. Goff (ed) From theory to practice; context in praxis. Selected papers from the $8^{\text {th }}$ Action Learning, Action Research World Congress Australia 2010, Action Learning Action Research Association, Toowong, Queensland, pp. 150-160.

Tacchi, J and Lennie, J 2014, 'A participatory framework for researching and evaluating communication for development and social change' In KG Wilkins, T Tufte \& R Obregon (eds) The handbook on development communication and social change. Wiley Blackwell, Oxford, pp. 298-320. 
UNDG 2011, Results-based management handbook, United Nations Development Group, October 2011.

Woodhill, J 2007, 'M\&E as learning: rethinking the dominant paradigm', in J de Graaff, J

Cameron, S Sombatpanit, C Pieri \& J Woodhill (eds) Monitoring and evaluation of soil conservation and watershed development projects, Enfield, USA, Science Publishers, pp. 83107. 